\section{Variability Analysis of Interconnect Structures Including General Nonlinear Elements in a SPICE-type Framework}

Alessandro Biondi, Paolo Manfredi, Dries Vande Ginste, Daniël De Zutter, Flavio Canavero.

In this paper, a stochastic modeling method is developed and implemented in a SPICE framework to analyze variability effects on interconnect structures including general nonlinear elements.

Introduction: Over the last few decades, computer-aided simulation tools have become an important asset for the design, simulation and optimization of complex electronic networks. Nonetheless, since large-scale integration and miniaturization lead to an important impact of the manufacturing process on the system performance, appropriate instruments are needed to evaluate uncertainties of the circuit parameters. Typical tools to gather quantitative statistical information of the circuit response are based on the well-know Monte Carlo (MC) method. This is computationally demanding, especially when realistic, complex structures are analyzed. Effective solutions to overcome these previous limitations have been proposed, leveraging the so-called polynomial chaos (PC) methods.

In [1], a PC-based technique, called the stochastic Galerkin method (SGM), was developed in Matlab for transmission lines terminated by linear loads. This technique for distributed circuits including linear elements was modified in [2] to allow implementation in a SPICE environment. A PC-method for lumped circuits consisting of discrete, linear and nonlinear elements was first reported in [3,4], allowing to model uncertainties in a small-signal regime or by approximating the nonlinearities by means of Taylor expansions. Recently, an improved PCtechnique for distributed circuit elements terminated by general nonlinear loads was conceived by the authors of the present contribution [5]. Unfortunately, this technique could solely be implemented in Matlab, as it relies on a finite-difference time-domain (FDTD) solver for transmission lines, making it also cumbersome to deal with lossy, dispersive lines and arbitrary circuit topologies. Therefore, in the present paper, a SPICE-compatible method is developed and implemented in a traditional environment allowing for the first time to perform PC-based variability analyses of lossy, dispersive multiconductor transmission lines terminated by general nonlinear loads.
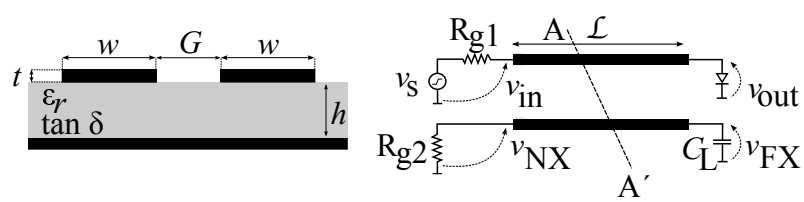

Fig. 1: Cross-section $A A^{\prime}$ (left) of the source-line-load configuration (right) of a pair of coupled microstrip lines.

Stochastic Modeling Formalism and Implementation: Consider a uniform multiconductor transmission line (MTL) with the propagation direction along the $z$-axis. The MTL consists of $\mathcal{N}$ signal conductors and a reference conductor. (An example of such a line is given in Fig. 1, where $\mathcal{N}=2$ ). Due to manufacturing, one or more geometrical and/or material parameters are not known in a deterministic way, thus they have to be treated as stochastic random variables (RVs), characterized by a probability density function (PDF), rendering the Telegrapher's equations nondeterministic. For ease of notation, in this section, we consider a single lossless dispersion-free line $(\mathcal{N}=1)$, affected by a single stochastic parameter $\beta$. We can then write the pertinent stochastic Telegrapher's equations as follows:

$$
\frac{\partial}{\partial z}\left[\begin{array}{c}
v(z, t, \beta) \\
i(z, t, \beta)
\end{array}\right]=-\left[\begin{array}{cc}
0 & L(\beta) \\
C(\beta) & 0
\end{array}\right] \cdot \frac{\partial}{\partial t}\left[\begin{array}{c}
v(z, t, \beta) \\
i(z, t, \beta)
\end{array}\right],
$$

where $v$ and $i$ are the voltage and current along the line, and with $L$ and $C$ the per-unit-of-length (p.u.1.) transmission line parameters. Next to the position $z$ along the line and the time $t$, we have also explicitly written down the dependence on the stochastic parameter $\beta$, of which only the PDF is known, hence prohibiting a straightforward solution of (1).
To solve (1), we rely on the SGM which is detailed in [1]. As a result of applying this method a novel set of deterministic Telegrapher's equations arises:

$$
\frac{\partial}{\partial z}\left[\begin{array}{c}
\tilde{\mathbf{v}}(z, t) \\
\tilde{\mathbf{i}}(z, t)
\end{array}\right]=-\left[\begin{array}{cc}
0 & \tilde{\overline{\mathbf{L}}} \\
\tilde{\mathbf{C}} & 0
\end{array}\right] \cdot \frac{\partial}{\partial t}\left[\begin{array}{c}
\tilde{\mathbf{v}}(z, t) \\
\tilde{\mathbf{i}}(z, t)
\end{array}\right] .
$$

The new unknowns $\tilde{\mathbf{v}}$ and $\tilde{\mathbf{i}}$ are $(K+1)$-vectors, containing voltage coefficients $\tilde{v}_{k}(z, t)$ and current coefficients $\tilde{i}_{k}(z, t)(k=0, \ldots, K)$. The parameter $K$ determines the number of terms in the so-called PCexpansions, as explained in [1]. $\tilde{\overline{\mathbf{L}}}$ and $\tilde{\overline{\mathbf{C}}}$ are known $(K+1) \times(K+1)$ p.u.l. matrices. The "augmented" system (2) is now fully deterministic, no longer showing dependence on $\beta$, and it can be implemented in a SPICE framework. Upon solving for $\tilde{\mathbf{v}}$ and $\tilde{\mathbf{i}}$, statistical information about $v$ and $i$ is readily obtained.

To solve the set of the $2(K+1)$ equations in (2), a set of proper $2(K+1)$ boundary conditions (BCs) is required. These are obtained by adding terminations to the lines. Assume that a nonlinear load is attached to the far-end terminal, i.e. at $z=\mathcal{L}$, with the following characteristic:

$$
i(\mathcal{L}, t, \beta)=F(v(\mathcal{L}, t, \beta)),
$$

where $F(\cdot)$ represents a general nonlinear function. Then, it was shown in [5] that by means of the SGM a new set of $K+1$ deterministic BCs is obtained, which can be cast in the form:

$$
\forall m=0, \ldots, K: \quad \tilde{i}_{m}(\mathcal{L}, t) \approx \sum_{q=1}^{Q} w_{m q}^{(1)} F\left(\sum_{k=0}^{K} w_{k q}^{(2)} \tilde{v}_{k}(\mathcal{L}, t)\right),
$$

where $w_{m q}^{(1)}$ and $w_{k q}^{(2)}$ are weights $(k, m=0, \ldots, K ; q=1, \ldots, Q)$ and $Q$ is a parameter that determines the accuracy. A similar set of BCs can be obtained at the near end $z=0$, for any kind of (non)linear load and generator. The novel BCs (4) connect all voltage and current expansion coefficients contained in $\tilde{\mathbf{v}}$ and $\tilde{\mathbf{i}}$ through the known nonlinear function $F(\cdot)$ and proper linear combinations. Hence, these deterministic BCs (4) are readily implemented in a SPICE framework, using dependent sources. This results in a somewhat more complex network in terms of number of nodes, but guarantees a very efficient simulation yielding comprehensive statistical information, and rendering this technique very useful for variability analysis during circuit design.

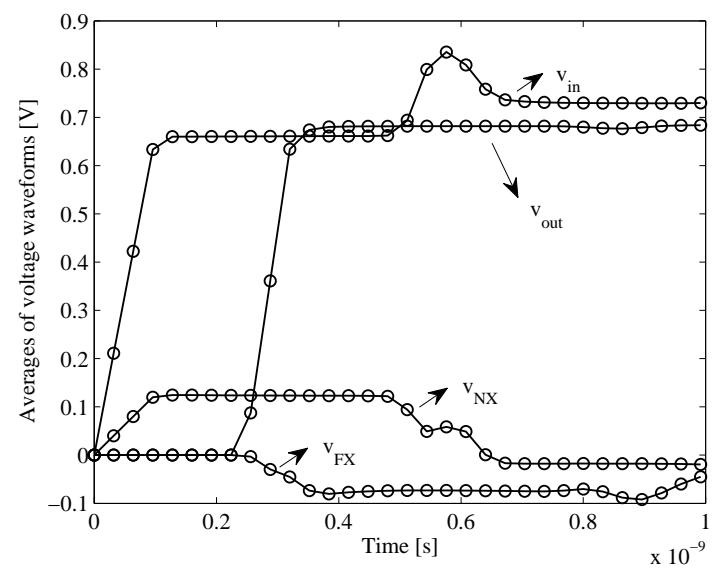

Fig. 2: Averages of the voltage waveforms $v_{\text {in }}(t), v_{\text {out }}(t), v_{\mathrm{NX}}(t)$, and $v_{\mathrm{FX}}(t)$, at the four terminals of the coupled microstrip lines of Fig.1. Circles (o): average computed using the SGM technique; full black lines: average computed using the $\mathrm{MC}$ technique.

Numerical Results: In this section the technique is validated by applying it to the variability analysis of the pair of coupled copper microstrip lines illustrated in Fig. 1. The length $\mathcal{L}$ is $5 \mathrm{~cm}$ and the gap $G$ between the lines and the relative permittivity $\epsilon_{r}$ of the substrate are considered to be two RVs uniformly distributed in the range [70,90] $\mu \mathrm{m}$ and $[3.7,4.3]$, respectively. The first line, i.e. the active line, is excited by means of a voltage source $v_{s}(t)$ that produces a ramped step, going from $0 \mathrm{~V}$ to $1 \mathrm{~V}$ in a risetime of $100 \mathrm{ps}$, in series with an impedance $R_{g 1}=50 \Omega$. This active line is terminated by means of a forward biased diode described by the 
well-known Shockley-model $i=I_{s}\left(e^{\frac{v}{\eta V_{t}}}-1\right)$, where $I_{s}=5 \cdot 10^{-14} \mathrm{~A}$, $\eta=1$, and $V_{t}=25.85 \mathrm{mV}$. The second line, called the victim line, is terminated at the near-end by a $50 \Omega$ load $R_{g 2}$. At the far-end, a $1 \mathrm{pF}$ ideal capacitor $C_{L}$ is connected. We chose to monitor the voltage waveforms $v_{\text {in }}$ at the input of the active line, $v_{\text {out }}$ at the diode, the near-end crosstalk $v_{\mathrm{NX}}$ and the far-end crosstalk $v_{\mathrm{FX}}$, all indicated on Fig. 1. The results are shown

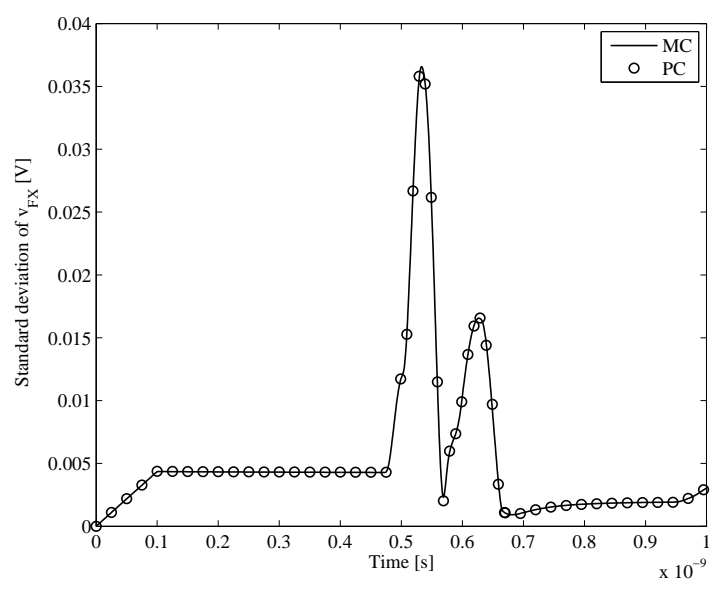

Fig. 3: Standard deviation of the far-end crosstalk $v_{\mathrm{FX}}(t)$. Circles (o): SGM technique; black line: MC technique.

in Fig. 2, where the continuous black lines represent the averages of the voltage waveforms obtained by performing a MC simulation in HSPICE, using 10000 samples of $G$ and $\epsilon_{r}$, drawn according to their respective uniform distributions. The proposed SGM approach for nonlinear loads was implemented and simulated also in HSPICE. The parameter $K$ was set to 5 and all losses, i.e. copper conductivity $\sigma=5.8 \times 10^{7} \mathrm{~S} / \mathrm{m}$ and substrate losses $\tan \delta=0.02$, were taken into account. The results of the proposed SGM are plotted as circles in Fig. 2. In Fig. 3 the standard deviation of $v_{\mathrm{FX}}(t)$ is presented. For both stochastic moments, i.e. average and standard deviation shown in Figs. 2 and 3, respectively, a very good agreement compared to the reference result is obtained.

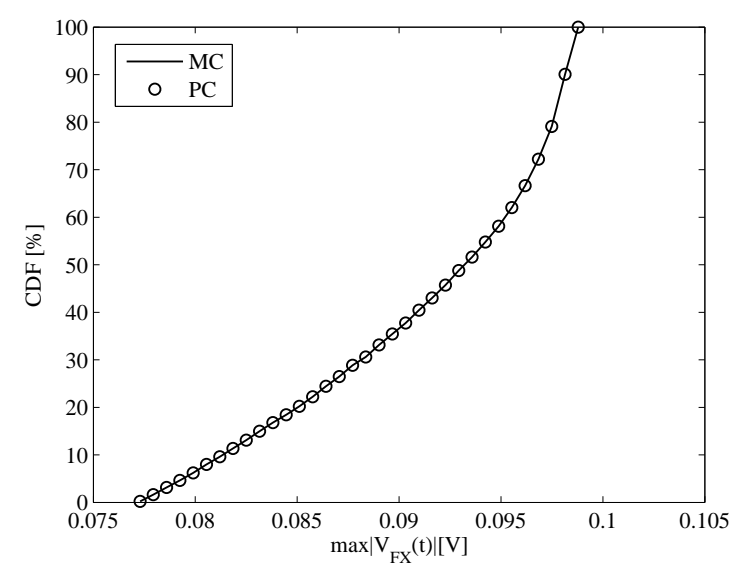

Fig. 4: $\mathrm{CDF}$ of the maximum of the far-end crosstalk for $v_{\mathrm{FX}}(t)$.

All computations have been performed using HSPICE on a Dell Latitude E6500 laptop with an Intel(R) Core(TM) 2 Duo T9900 CPU running at $3.06 \mathrm{GHz}$ and $4 \mathrm{~GB}$ of RAM. The total runtime for the $\mathrm{MC}$ analysis was $5656.4 \mathrm{~s}$, the SGM simulation only took $1.11 \mathrm{~s}$. A remarkable speed-up factor exceeding 5000 is obtained by means of the SGM approach.

Usually, designers are interested in quantifying the maximum amount of crosstalk that can be expected from a certain topology. Therefore, in a postprocessing step and in addition to stochastic moments, we compute the cumulative distribution function (CDF) of the maximum far-end crosstalk, $\max _{t \geq 0}\left|v_{\mathrm{FX}}(t)\right|$. From Fig. 4, an excellent agreement between the MC simulations and the novel approach is again observed.
Conclusion: A stochastic method was developed and implemented in a SPICE-type framework for the variability analyses of lossy, dispersive MTLs in the presence of general nonlinear loads. The methodology was validated and illustrated in the HSPICE environment by means of an example consisting of a pair of coupled microstrip lines exhibiting variability of its geometrical and material parameters, terminated by a nonlinear diode. Compared to the standard Monte Carlo analysis, this method delivers an excellent agreement and shows superior efficiency.

Alessandro Biondi, Dries Vande Ginste, Daniël De Zutter ( Department of Information Technology-INTEC, Ghent University, Ghent, Belgium)

E-mail: alessandro.biondi@ugent.be

Paolo Manfredi, F. G. Canavero ( Department of Electronics, Polytechnic of Turin, Turin, Italy)

\section{References}

1 D. Vande Ginste, D. De Zutter, D. Deschrijver, T. Dhaene, P. Manfredi and F. G. Canavero, "Stochastic modeling based variability analisys of onchip interconnects", IEEE Trans. on Components, Packaging and Manufacturing Technology, vol. 2, pp. 1182-1192, Jul. 2012.

2 P. Manfredi, D. Vande Ginste, D. De Zutter and F. G. Canavero, "Uncertainty assessment of lossy and dispersive lines in SPICE-type environments", IEEE Trans. on Components, Packaging and Manufacturing Technology, vol. 3, pp. 1252-1258, Jul. 2013.

3 Q. Su and K. Strunz, "Stochastic circuit modelling with Hermite polynomial chaos", Electronic Letters, vol. 41, pp. 1163-1165, Oct. 2005.

4 K. Strunz and Q. Su, "Stochastic formulation of SPICE-type electronic circuit simulation with polynomial chaos", ACM Trans. on Modeling and Computer Simulation, vol. 18, pp.15:1-15:23, Sep. 2008.

5 A. Biondi, D. Vande Ginste, D. Dezutter, P. Manfredi and F. G. Canavero, "Variability analysis of interconnects terminated by general nonlinear loads", IEEE Trans. on Components, Packaging and Manufacturing Technology, vol. 3, pp. 1241-1251, Jul. 2013. 